\title{
„Ich würde ja gerne Medien einsetzen, aber ich habe kein Smartboard im Klassenraum!“ Eine Untersuchung des Einsatzes von neuen Medien im Russischunterricht an einer Gesamtschule
}

\begin{abstract}
Against the background of the process of digitization in German schools, the present study examines the use of digital media in Russian lessons as a function of three core factors: media literacy, teacher motivation, and school equipment. After several weeks of observing Russian lessons, a guided interview was conducted with two teachers. The evaluation of the overall results shows that the three factors mentioned interact with one another and, depending on the situation, can either have a beneficial or inhibiting effect on each other. While lack of equipment can lower teachers' motivation, their affinity to new media and initiative can outweigh the material constraints. Afterwards, suggestions will be presented on how digital media can be meaningfully used in Russian lessons if the school's facilities offer few opportunities.
\end{abstract}

На фоне процесса дигитализации немецких школ в данном исследовании рассматривается использование цифровых средств массовой информации на уроках русского языка в зависимости от трех основных факторов: медиаграмотности и мотивации преподавателя, а также оснащения школ. После нескольких недель наблюдения на уроках было проведено интервью с двумя учителями русского языка. Исследования показывают, что все три фактора взаимодействуют друг с другом и, в зависимости от ситуации, могут оказывать как полезное, так и негативное действие друг на друга. С одной стороны, недостаточная материальная база в школе может ослабить мотивацию учителей, в то же время открытость к новым

Anastasia Drackert und Katrin Bente Karl (Hg.), Didaktik der slawischen Sprachen

Beiträge zum 2. Arbeitskreis in Innsbruck (19.02.-20.02.2018)

(C) 2019 innsbruck university press, ISBN 978-3-903187-80-1, DOI 10.15203/3187-80-1 
средствам и инициатива могут компенсировать этот дефицит. Далее представлены предложения, какиим образом дигитальные средства могут быть более целенаправленно использованы на уроках русского языка, если технические возможности школ ограничены.

\section{Einleitung}

Die Meinung, dass Schulunterricht auf digitale Medien angewiesen sei, ist in der heutigen Zeit stark verbreitet und wird - wahrscheinlich - von den meisten MitbürgerInnen vertreten. Schließlich leben wir in einer Welt, die ohne digitale Inhalte und Werkzeuge nicht mehr oder nur eingeschränkt funktionsfähig ist. So wird von SchulabsolventInnen erwartet, dass sie bereits vor dem Berufseinstieg über grundlegende Kenntnisse in der elektronischen Datenverarbeitung verfügen. Bewerbungsverfahren für einen Job oder Studienplatz werden meist elektronisch durchgeführt und bei Bewerbungsgesprächen werden häufig Video-Messaging-Programme eingesetzt.

Digitalisierung an Schulen ist also nicht nur ein Forschungsfeld für die Schuldidaktik, sondern auch ein in der Gesellschaft häufig diskutiertes Thema. Die Relevanz und das Interesse der Öffentlichkeit können an der Vielzahl von Zeitungsartikeln, Meinungen und Kommentaren eingeschätzt werden. In deutschen Printmedien wurden die unterschiedlichen Positionen diesbezüglich häufig aufgegriffen und haben meist ein Bild skizziert, das auf Defizite im Schulsystem hinweist: „Wie Schulen sich für digitale Zukunft ändern müssen“ (Tagesspiegel, 12.04.2018), „Fehler 404'“ (Zeit Online, 31.01.2018), „Technik hat den Menschen zu dienen“

\footnotetext{
${ }^{1}$ Fehler 404: Eine Fehlermeldung, die ausgegeben wird, wenn der gesuchte Inhalt (z.B. angeforderte Internetseite) online nicht erreichbar ist. Hier bezieht sich die Meldung auf das Fehlen von nötigen Technologien an deutschen Schulen.
} 
(Süddeutsche Zeitung, 24.02.2018) und „Wenn Lehrer mit der Internetverbindung kämpfen“" (Frankfurter Allgemeine, 14.01.2017).

Trotz bekannter technischer Mängel an vielen deutschen Schulen wird von der Lehrperson erwartet, dass sie zur Vermittlung des Unterrichtsstoffes nicht nur das Lehrbuch und die Tafel nutzt, sondern darüber hinaus u.a. mit Darstellungen auf einem Tageslichtprojektor arbeitet, das Internet zu Recherchezwecken einsetzt und authentische Hörspiele und Videos vorführt.

Medienkompetenz - also der korrekte und kritische Umgang bzw. Einsatz von Medien - ist daher ein wichtiger Punkt im Kernlehrplan für das Fach Russisch in Nordrhein-Westfalen (KLP 2014: 16). Hier wird der mediale Aspekt nicht nur in dem Abschnitt „Text- und Medienkompetenz“ behandelt, sondern auch in den Bereichen der Sprachlern- und interkulturellen kommunikativen Kompetenzen. Für SchülerInnen und für LehrerInnen ist es daher gleichermaßen wichtig, mediale Kompetenzen zu erwerben.

Das Ziel der vorliegenden Arbeit besteht darin herauszufinden, wie sich Einstellung und Fähigkeit der LehrerInnen auf den Einsatz digitaler Medien in ihrem Unterricht auswirken. Dafür soll untersucht werden, welche hemmenden und förderlichen Faktoren beim Einsatz digitaler Medien im Russischunterricht eine Rolle spielen. Denn im Gegensatz zu anderen Unterrichtsfächern fehlt es für das Fach Russisch noch an empirischen Untersuchungen zum Einsatz digitaler Medien in der Praxis.

Zunächst werde ich auf das Themenfeld Medien eingehen. Dabei stelle ich kurz dar, was unter diesem Begriff im schulischen Kontext fällt. Im Folgenden gehe ich auf die Medienkompetenzen sowohl von SchülerInnen als auch von LehrerInnen ein, um anschließend die Diskussion über die Sinnhaftigkeit bzw. Notwendigkeit des Einsatzes digitaler Medien an deutschen Schulen im Fremdsprachenunterricht aufzugreifen. Nach der theo-

Anastasia Drackert und Katrin Bente Karl (Hg.), Didaktik der slawischen Sprachen

Beiträge zum 2. Arbeitskreis in Innsbruck (19.02.-20.02.2018)

(C) 2019 innsbruck university press, ISBN 978-3-903187-80-1, DOI 10.15203/3187-80-1 
retischen Aufarbeitung des Themas präsentiere ich meine eigene, praxisnahe Forschung zum Einsatz digitaler Medien. Zunächst erfolgt eine Auswertung des Russischunterrichtes im Hinblick auf die Art und Weise des Medieneinsatzes. Dem folgt ein Interview mit Russischlehrkräften sowie die Deutung der Forschungsergebnisse im Bezug aufeinander. Schließlich möchte ich darstellen, wie bereits mit schwacher technischer Ausstattung der Schule, neue Medien den analogen Russischunterricht sinnvoll erweitern können.

\section{Medien - Definition und Kategorisierung}

„Medien können ganz allgemein als Vermittlungsträger von Informationen verstanden werden“, so der erste Satz zum Begriff „Medien“ im Metzler Lexikon für Fremdsprachendidaktik (Grünewald 2010: 207). Dieser Definition nach muss ein Medium nicht nur Informationen enthalten, sondern diese auch dem Nutzer des Mediums vermitteln. Der Begriff Medium ist nicht an bestimmte Objekte geknüpft, er beschreibt vielmehr ein unbestimmtes Objekt, durch das kommunikationsbezogene Absichten verfolgt werden. Als Beispiel behaupten Grafe, Herzig und Tulodziecki (Grafe, Herzig, Tulodziecki 2010: 32), dass ein Buch an sich noch kein Medium darstelle. Erst wenn dieses Buch im kommunikativen Zusammenhang stünde, sei es ein Medium. Das erklärt, warum der Begriff Medium für unterschiedliche Sachverhalte verwendet wird, wie für Tafel und Kreide, aber auch für Spielfilm und Computerspiel. Ein Medium ist also ein Objekt, oder ein Vorgang, durch das Informationen bzw. Sachverhalte vermittelt werden können. 


\subsection{Kategorisierung der Medien}

Sachverhalte können in unterschiedlicher Form erlernt bzw. erfahren werden. Zusammengefasst nach Grafe, Herzig und Tulodziecki ergeben sich folgende Kategorien (2010: 29):

1. In der realen Form wird ein unmittelbarer Kontakt in der Wirklichkeit hergestellt, z.B. beim Beobachten oder Interagieren.

2. Bei der modellhaften Form wird Information durch modellhafte Nachbildungen vermittelt.

3. In der abbildhaften Form werden Informationen mit Hilfe von objektgetreuen Darstellungen überbracht.

4. Bei der symbolischen Form handelt es sich um eine Vermittlung durch verbale Darstellungen oder nicht-verbale Zeichen.

Zum besseren Verständnis kann man die vier Formen auf ein Beispiel übertragen: wenn ein Kind den Sachverhalt „Zoo“ kennenlernt bzw. erlebt, in dem es den Ort besucht, dann handelt es sich um die reale Form der Informationsvermittlung. Wenn das Kind den Zoo nicht direkt erlebt, sondern beispielsweise durch das Spielen mit Playmobil Tierfiguren, dann handelt es sich um die modellhafte Form. Bereits bei dieser Form findet ein großer Informationsverlust statt, weil nicht die gesamte Wirklichkeit abgebildet bzw. übermittelt wird. Die abbildhafte Form wäre bei diesem Beispiel eine Zeichnung oder eine Dokumentation über den Zoo. Schließlich wäre eine Erzählung, ohne bildhafte Unterstützung, die symbolische Form der Vermittlung. Daraus kann man schließen, dass je weiter die Form von der Wirklichkeit abweicht, desto schwieriger es für das Kind ist die Wirklichkeit nachzuvollziehen. Für den Schulunterricht bedeutet diese Tatsache, dass trotz des Medieneinsatzes zwischen SchülerInnen und der Wirklichkeit eine gewisse Distanz bestehen bleibt. So werden im Russischunterricht SchülerInnen, die den Kreml nur auf Fotografien kennengelernt haben, nicht den gleichen Bezug herstellen, wie SchülerInnen, die 
vor Ort gewesen sind, was beim Vermitteln von landeskundlichen Inhalten von der Lehrkraft beachtet werden soll.

Medien lassen sich noch weiter kategorisieren als nur nach ihrer Vermittlungsform. So gliedert Wapenhans Medien in vier weitere Kategorien und Unterkategorien (Wapenhans 2014: 254-255). Zuerst unterscheidet sie Medien nach dem Grad der Modernität. Dazu gehört die Steigerung von nicht technischen Medien wie Bilder, Landkarten, Lektüren und Schautafeln bis zu den modernen und neuen Medien wie Film und Internet. An dieser Stelle muss allerdings ergänzt werden, dass die Unterkategorien sich überkreuzen können. So gehört zwar nach dieser Definition eine Landkarte zum untersten Grad der Modernität, allerdings lässt sich eine Karte mittlerweile auch im Internet betrachten. Weiter werden Medien nach dem Grad des Technikeinsatzes unterschieden. So gehören Menschen (z.B. Lehrpersonen) zu den Primärmedien. Wird Information durch Schreib- und Druckmedien vermittelt, spricht Wapenhans (2014: 254) von Sekundärmedien. Dazu gehören unter anderem Bilder und Schulbücher. $\mathrm{Zu}$ den Tertiärmedien gehören elektronische Medien wie z.B. Fernsehen und Radio. Und schließlich die Quartärmedien, also digitale Medien, die Interaktivität ermöglichen. Dazu gehören Internet als Informationsquelle sowie Chat und E-Mail. Die dritte Kategorie ist die Unterscheidung nach dem Wahrnehmungskanal, also die Art und Weise der Informationsaufnahme durch einen Rezipienten. Dazu gehören Medien, die visuell (Informationsvermittlung über das Auge), auditiv (über das Ohr), audiovisuell (Kombination aus Auge und Ohr) sowie multimodal (über mehrere Sinnesorgane, z.B. am PC) Inhalte übermitteln. Zuletzt nennt Wapenhans (2014: 254 f) den Grad der Didaktisierung der Medien. Nicht didaktisierte, authentische Medien sind im Fremdsprachenunterricht in erster Linie an MuttersprachlerInnen gerichtet. Dabei handelt es sich um Medien in ihrer Originalfassung ohne Bearbeitung, wie z.B. Blogs, Filme und Internetseiten. Wurden die Medien zu didaktischen Zwecken erstellt oder bearbeitet, so spricht man von Didaktisierung. Dazu zählen Medien für Fremdsprachen-LernerInnen wie z.B. Schulbuchtexte und Grammatik- 
Übersichten. Die letzte Unterkategorie umfasst didaktisierte, authentische Medien, die für Lehrzwecke bearbeitet wurden, wie z.B. gekürzte und kommentierte literarische Texte.

Zusammenfassend kann man auf die Definition bzw. Eingrenzung des Medienbegriffs von Grafe, Herzig und Tulodziecki zurückgreifen: „Der Begriff Medien sowie die Begriffe Medienpädagogik, Mediendidaktik und Medienerziehung sind im Kontext der sich ausbreitenden technischen Vermittlungsmöglichkeiten von Inhalten durch Film, Radio und Fernsehen entstanden und bezüglich ihrer Begriffsinhalte weiterentwickelt worden“ (Grafe, Herzig \& Tulodziecki 2010: 31). Der Begriff Medien beschreibt daher Vermittler von Informationen mit technischer Unterstützung.

\subsection{Medienkompetenz von LehrerInnen und SchülerInnen im Schul- unterricht}

Medienkompetenz ist fächerübergreifend ein fester Bestandteil des Kernlehrplans für Nordrhein-Westfalen. So heißt es beispielsweise, dass SchülerInnen, ,[...] ein grundlegendes Spektrum an Hilfsmitteln und Medien funktional nutzen, um eigene Texte in mündlicher wie schriftlicher Vermittlungsform adressatenorientiert zu stürzen" (KLP 2014: 24). Weiterhin heißt es im Abschnitt für interkulturelle kommunikative Kompetenz: „Die Schülerinnen und Schüler können in interkulturellen, sprachlich auch anspruchsvollen Kommunikationssituationen sowohl in direkten persönlichen Begegnungen als auch im Umgang mit russischsprachigen Texten und Medien handeln“ (KLP 2014: 30). Danach bedeutet dies für SchülerInnen zunächst, dass sie Medien für die Textproduktion einsetzen müssen. Zum anderem müssen sie mit fremden Medien reflektiert und kritisch umgehen können. 
Spanhel erläutert die Anforderungen an SchülerInnen detaillierter. Zusammengefasst ergeben sich so folgende Punkte, die SchülerInnen beherrschen müssen (Spanhel 2001: 268):

1. Auswählen und Nutzen von Medienangeboten unter Abwägung von Handlungsalternativen

2. Eigenes Gestalten und Verbreiten von Medienbeiträgen

3. Verstehen und Bewerten von Mediengestaltungen

4. Erkennen und Aufarbeiten von Medieneinflüssen

5. Durchschauen und Beurteilen von Bedingungen der Medienproduktion und Medienverbreitung.

Die Aufgabe der Lehrperson ist daher nicht nur das Lehren von Medienumgang, sondern auch die Aufstellung eines breiten Medienspektrums und Förderung von Gestaltung medialer Beiträge durch SchülerInnen.

Um SchülerInnen Inhalte vermitteln zu können, müssen Lehrkräfte selbst Sachkenntnis über diese Inhalte erlangen. Daher müssen auch LehrerInnen Medienkompetenzen erwerben und sich stets weiterbilden, um mit neuen Medien umgehen zu können. Zusammengefasst gehören zu den Fähigkeiten, über die Lehrkräfte verfügen sollten (ebd.):

1. Die Bedeutung von Medien für Kinder und Jugendliche zu erfassen und im Fachunterricht zu berücksichtigen

2. Die Medienangebote im Unterricht in reflektierter Weise zu verwenden

3. Medienthemen in angemessener Form zu behandeln

4. In der Schule personale und institutionelle Bedingungen für medienpädagogische Umsetzungen zu durchschauen.

Medieneinsatz im Unterricht erfordert also eine vorausgehende Planung. Wenn Inhalte mit Medien vermittelt werden sollen, muss die Lehrkraft überlegen, welche Methode der Präsentation und welche Art von Medien 
am sinnvollsten wären. Schließlich sollen Medien SchülerInnen auf dem Weg zum Unterrichtsziel unterstützen und nicht das Ziel des Unterrichts sein.

\section{Digitaler Unterricht an der Schule}

Wie Hartmann und Purz ausführen, ist „,der Einsatz digitaler Medien im Unterricht unabdingbar, um Kindern und Jugendlichen die aktive Partizipation und kritische Hinterfragung moderner Gesellschaftsformen zu ermöglichen. [...] Das Ziel für Lehrerinnen und Lehrer muss sein, einen Unterricht zu gestalten, der sowohl analoge als auch digitale Kompetenzen, die Kinder und Jugendliche in der Wissensgesellschaft zur Teilhabe befähigen sollen, zu forcieren und die Selektion, Rezeption, Produktion und kritische Reflexion dieser Medien zu einem selbstverständlichen Teil des Unterrichts zu machen“" (2018: 142). Doch ebenso stellt sich die Frage, ob Schule als Institution in der Lage ist, digitale Inhalte sinnvoll zu vermitteln und ob es generell der richtige Ort ist für Inhalte und Methoden, die aus dem Blick der Bildungswissenschaft kritische Merkmale aufweisen. Im Folgenden möchte ich auf einige wesentliche Argumente für und gegen digitale Medien im Unterricht eingehen.

\subsection{Gehören digitale Medien in die Schule? Über den Sinn des digita- len Lernens}

Fakt ist, dass digitale Medien den Alltag der SchülerInnen erobert haben. Ein Musik-Clip oder sogenanntes Tutorial bei YouTube, ein „Like“ bei Instagram für ein Urlaubsfoto von Freunden und die Hausaufgaben in 
einer klasseninternen WhatsApp-Gruppe - so könnte eine Sequenz aus dem Tag vieler durchschnittlicher SchülerInnen aussehen. Doch auch das Nutzen von GeoGebra für eine Geometrie-Aufgabe, das Fremdsprachenlernen mit einer Serie, die in der Originalsprache ausgestrahlt wird und das Führen eines digitalen Portfolios gehören zum Alltag vieler SchülerInnen.

Heinz Moser (2014: 9 f) nutzt den Begriff „Digital Natives“, um die Generation der Menschen zu bezeichnen, die mit den neuen Medien aufgewachsen sind und wie selbstverständlich damit umgehen. Dadurch, dass Medien für diese Gruppe stets omnipräsent sind, haben „Digital Natives“ ein angepasstes Lern- und Konsumverhalten. Für sie stellt sich nicht die Frage, ob der Einsatz digitaler Medien und Methoden sinnvoll ist, da diese bereits zu ihrem Alltagsleben gehören. Trifft dieser digitale Alltag auf die analoge Schule, können Diskrepanzen entstehen. Lehrkräfte sehen in Geräten, die den Zugang zu digitalen Inhalten ermöglichen, zunächst einmal eine weitere Störungsquelle im Unterricht. Daher haben viele Schulen ein permanentes Verbot von Smartphones auf dem Schulgelände.

Weiter wird von Moser die Frage aufgeworfen, ob die Schule es sich überhaupt leisten kann, in der globalisierten Welt auf den Einsatz digitaler Medien zu verzichten. Im deutschen Schulgesetz heißt es: „Schülerinnen und Schüler werden befähigt, verantwortlich am sozialen, gesellschaftlichen, wirtschaftlichen, beruflichen, kulturellen und politischen Leben teilzunehmen und ihr eigenes Leben zu gestalten“" (Schulgesetz 2005: §2). Werden digitale Medien und Werkzeuge aus der Schule verbannt oder nur oberflächlich behandelt, besteht die Gefahr, dass SchülerInnen den richtigen Umgang nicht lernen. Daher muss die Schule die Nutzung neuer Medien als eine Chance begreifen, Kinder und Jugendliche kompetent zu unterstützen.

Nach Heinz Moser können Lehrkräfte sich auf folgende drei Aspekte stützen (Moser 2014: 10): 
1. Digitale Medien eignen sich ideal als Wissensressourcen im Unterricht. Die korrekte Arbeitsweise muss jedoch von der Lehrkraft gelehrt werden. SchülerInnen sind zwar oft kompetent im Umgang mit Medien, wenn es um reine Unterhaltung geht, jedoch fehlt ihnen die Herangehensweise an digitale Medien und Werkzeuge zum wissenschaftlichen Arbeiten.

2. Des Weiteren fehlt den „Digital Natives“ oft der verantwortungsvolle Umgang mit digitalen Medien. Dadurch, dass sie Medien als etwas Selbstverständliches und Alltägliches wahrnehmen, fehlt SchülerInnen ein ,gesunder Abstand“, um reflektiert die Mediennutzung zu hinterfragen (z.B. Privatsphäre, Sicherheit, Glaubwürdigkeit). Natürlich kann Schule nur schwer mit allen aktuellen technologischen Entwicklungen und ihren Risiken mithalten bzw. diese überblicken, doch kann hier die richtige Grundeinstellung vermittelt werden.

3. Schließlich können LehrerInnen positive Grundeinstellung und Affinität, die SchülerInnen gegenüber digitalen Medien haben, für den eigenen Unterricht nutzen. So könnten Fremdsprachenlehrkräfte beispielsweise echte (Video-)Blogs im Unterricht nutzen, weil Jugendliche diese auch in ihrer Freizeit konsumieren.

Manfred Spitzer kommt jedoch zu einem anderen Ergebnis: „Nach den vorliegenden wissenschaftlichen Erkenntnissen braucht man einen Computer zum Lernen genauso dringend wie ein Fahrrad zum Schwimmen oder Röntgengerät, um Schuhe anzuprobieren“" (Spitzer 2012: 94). Spitzers Studien nutzt Ralf Lankau (2017) in seinem Werk „Kein Mensch lernt digital", um zu belegen, dass das Lernen ein analoger Prozess ist, der von Nutzung digitaler Medien und Werkzeuge eher gestört als gefördert wird. Seiner Meinung nach muss der Lernort Schule möglichst digitalfrei bleiben bzw. werden. Lankau erkennt zwar die Vorteile einer digitalisierten Lernkultur, benennt aber gleichzeitig die dadurch entstandenen Probleme. 
So beeinflussen sich die Lesekompetenz und Mediennutzung von SchülerInnen gegenseitig. Kinder und Jugendliche, die mehr Medien konsumieren, weisen öfter Defizite beim Lesen auf (ebd. 128 ff). Sind es Kinder aus sozialschwachen Familien, vergrößert sich das Dilemma: „Kinder aus benachteiligten Schichten haben um einen Faktor drei mehr Fernsehen und Faktor vier mehr Spielkonsolen im eigenen Zimmer. Dies hat dramatische, durch die Medienwirkungsforschung gut belegte, Auswirkungen. Nachgewiesen ist ein erhöhtes Risiko für Verzögerungen in der Sprach- und Bewegungsentwicklung, für Übergewicht, für Schlafstörungen, für Empathieverlust, und für Schulversagen" (Bleckmann 2016: 2). Es stellt sich also die Frage, ob SchülerInnen in der Schule regelmäßig mit digitalen Medien arbeiten sollen, wenn sie ihre analogen Fähigkeiten noch nicht erlernt haben. Auch die Annahme, dass SchülerInnen den neuen Medien gegenüber eine positive Grundeinstellung haben, wird von Lankau hinterfragt. Nach der Studie des Wissenschaftszentrum Berlin lehnen knapp die Hälfte der Jugendlichen eine frühe Digitalisierung ab: „Jugendliche verbringen täglich mehrere Stunden mit ihrem Smartphone. Trotzdem - oder vielleicht gerade deshalb - sind sie skeptischer als ihre Eltern, digitalaffine Junglehrer oder Bildungspolitiker" (Lankau 2017: 137).

\subsection{Digitale Medien im Zusammenspiel mit analogem Unterricht}

Digitale Medien im Unterricht können also nur dann sinnvoll eingesetzt werden, wenn sie den analogen Unterricht nicht hindern, sondern bereichern. Für die Praxis würde das bedeuten, dass zum Beispiel der Einsatz einer Internetrecherche nicht den Diskurs zwischen LehrerInnen und SchülerInnen ersetzen oder behindern darf, sondern diesen sinnvoll unterstützt, indem man die Recherche nur in der Erarbeitungsphase nutzt. 
Das pädagogische Dreieck beschreibt die Beziehung des Inhalts (also des Unterrichtsfaches), des Lernenden und des Lehrenden zueinander. In diesem System findet zwischen den beiden Akteuren ein direkter Austausch statt, wodurch der Inhalt erarbeitet wird. Lehrmedien, analoge sowie digitale, dienen als Werkzeuge zur Vermittlung des Wissens über den Inhalt. Lankau (2017: 44) nimmt aus dem pädagogischen Dreieck den Lehrenden heraus bzw. ersetzt diesen durch digitale Medien (siehe Abb. 1).
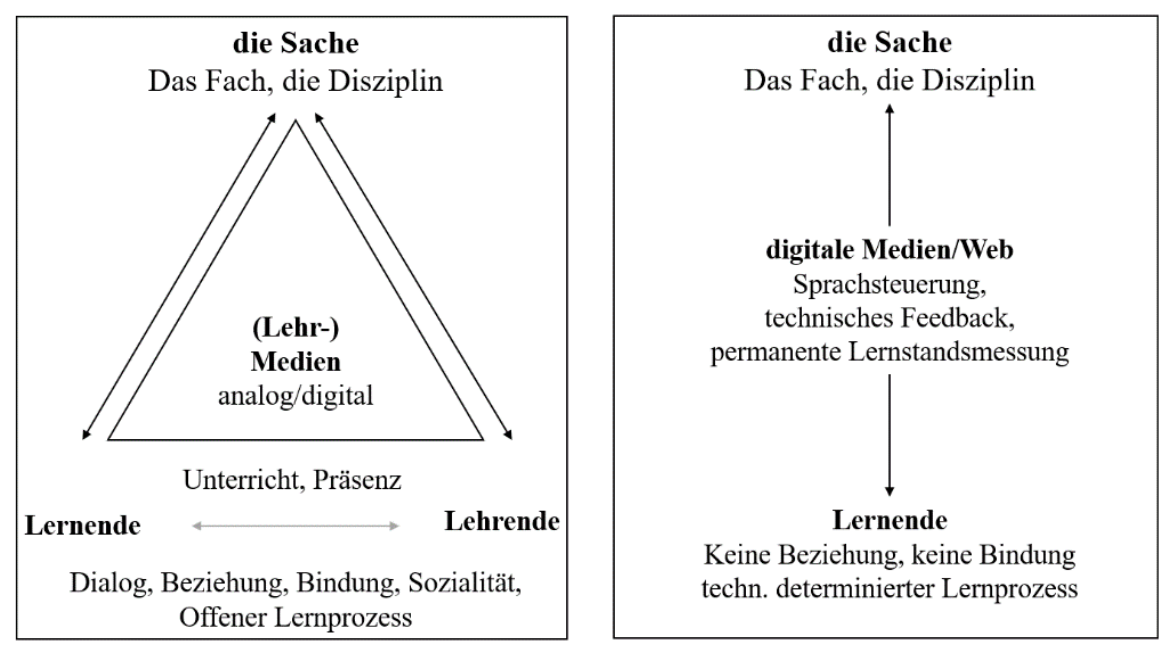

Abb. 1 Das pädagogische Dreieck nach Lankau (2017, S. 44)

Übrig bleibt die Sache, also der Lerninhalt, und digitale Medien, als Wissensquelle und Arbeitswerkzeug. Dadurch, dass die Lehrkraft nicht mehr den Lernprozess steuert und zwischen ihr und den SchülerInnen kein Dia$\log$ entsteht, entwickeln SchülerInnen keine Beziehung und Bindung zu dem Fach. Zwar handelt es sich dabei nach wie vor um eine Form des Lernens, guter Unterricht ist es jedoch nicht mehr. 
Demgegenüber zeigt Heinz Moser (2014: 48), wie ein guter Unterricht im digitalen Zeitalter aussehen könnte. Auch er greift dabei auf das pädagogische Dreieck zurück, erweitert es jedoch um ein weiteres Dreieck - ein Mediendreieck (siehe Abb. 2).

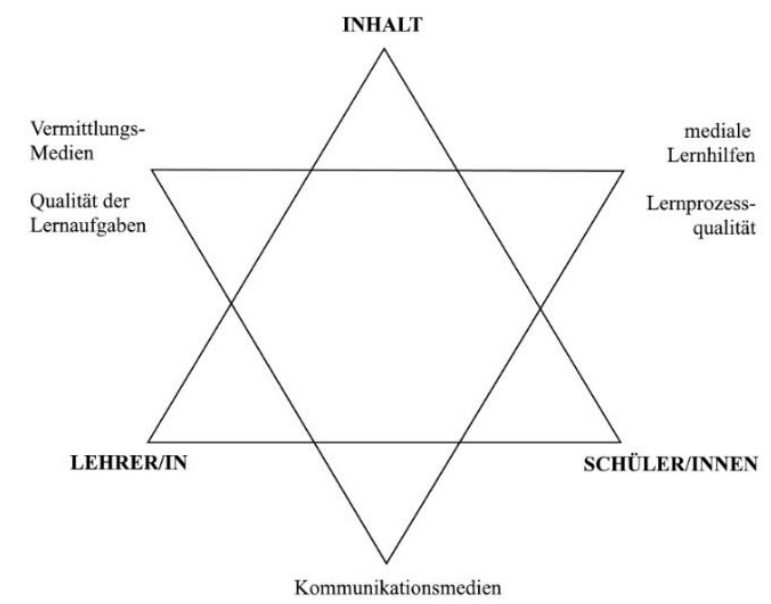

Abb. 2 Das pädagogische Sechseck nach Moser (2014, S. 48)

In diesem Modell werden keine Akteure des Unterrichts ersetzt. Der klassische, analoge Unterricht wird mit Hilfe von digitalen Medien angereichert und steigert die Qualität der Lernaufgaben und des Lernprozesses. Auf der einen Seite stehen der Lehrkraft durch diese Erweiterung mehr Werkzeuge zur Verfügung. Auf der anderen Seite profitieren die SchülerInnen von digitalen Lernhilfen und einem abwechslungsreicheren Unterricht. Eigenständig können digitale Medien also nur eingeschränkt neue Inhalte vermitteln bzw. aufarbeiten. Ein lehrender Akteur wird zwingend vorausgesetzt, damit eine Bindung zwischen den Lernenden und dem Unterrichtsinhalt aufgebaut werden kann. Nutzt die Lehrperson digitale Medien, kann der Unterricht qualitativ aufgewertet werden. 


\subsection{Mögliche Hindernisse bei der Umsetzung der Schuldigitalisierung}

In der Schulrealität hat sich die Meinung durchgesetzt, dass digitale Medien auf dem Lehrplan stehen sollten: „Digitalisierung ist eines der Kernthemen der Landesregierung" (www.schulministerium.nrw.de 2018). Daher stellt sich die Frage, warum die Digitalisierung des Unterrichts nur schleppend vorankommt.

Moser (2014: 14-18) benennt sechs Ursachen für die träge Entwicklung, die im Folgenden zusammengefasst werden:

1. Ohne die geeignete Ausstattung können digitale Medien nicht im Unterricht eingesetzt werden. Zwar hat sich an den deutschen Schulen die Ausstattung der Lernräume deutlich verbessert, trotzdem stehen Lehrkräfte immer noch vor Hürden, wenn sie die Technik im Unterricht einsetzen (z.B. Reservierung des Computerraumes, veraltete Systeme, inkompatible Geräte usw.). Unerwähnt darf außerdem nicht bleiben, dass gute Ausstattung nicht gleichzusetzen ist mit gutem oder häufigem Einsatz von digitalen Medien.

2. Die Weiterentwicklung der Hardware schränkt den Einsatz im Unterricht ebenfalls ein. Wurden früher Computerräume gefordert, so sind es heute eher Tablets und E-Books, die sich flexibler im Unterricht einsetzen lassen. Außerdem verfügen viele SchülerInnen über Smartphones, die sich ebenfalls im Unterricht einsetzen lassen und den alten PCs in manchen Einsatzszenarios überlegen sind. Aus diesen Gründen ist es nachvollziehbar, dass die Ausstattung der Schulen mit neuer Hardware eine komplexe Aufgabe darstellt. 
3. Medialer Habitus der Lehrkräfte könnte ein weiterer Grund für die schleppende Digitalisierung darstellen. Diverse Untersuchungen belegen, dass Lehrkräfte eher kritisch den neuen Medien gegenüberstehen, da diese eher mit bildungsirrelevanten Inhalten in Verbindung gebracht werden (Biermann 2009; Kommer 2010).

4. Vielen LehrerInnen fehlt es an mediendidaktischem Wissen. Auch wenn Technik zur Verfügung steht und Lehrkräfte die Vorteile von digitalen Medien kennen, wissen viele von ihnen nicht, wie sie den Unterricht mit Medien anreichern, ohne den Unterrichtsfluss zu stören. Digitalisierung des Unterrichts kann erst dann erfolgen, wenn es den analogen Unterricht nicht hindert, sondern bereichert.

5. Unter strukturellen Gründen versteht Moser die rasante Medienentwicklung und bildungspolitischen Entscheidungen als Reaktion auf eben diese Entwicklung. Stand in den 80er Jahren hauptsächlich das Programmieren an einem PC im Vordergrund, so stehen heute Themen wie Internet als Informationsquelle und verantwortungsvolle Mediennutzung auf dem Plan.

6. Schließlich ist der Lehrplan bzw. seine Aktualität bzgl. der Medienbildung einer der Gründe für die ablehnende Haltung gegenüber neuen Medien. Digitale Inhalte werden als ein Werkzeug aller Fächer betrachtet, ohne dass sie eine größere und dauerhafte Beachtung finden.

Um dieser Entwicklung entgegenzuwirken, beschloss die Kultusministerkonferenz vom 8. März 2012 Folgendes (KMK 2012: 3): „Schulische Medienbildung versteht sich als dauerhafter, pädagogisch strukturierter und begleiteter Prozess der konstruktiven und kritischen Auseinandersetzung mit der Medienwelt. Sie zielt auf den Erwerb und die fortlaufende Erwei- 
terung von Medienkompetenz, also jener Kenntnisse, Fähigkeiten und Fertigkeiten, die ein sachgerechtes, selbstbestimmtes, kreatives und sozial verantwortliches Handeln in der medial geprägten Lebenswelt ermöglichen."

Wie lässt sich das bisher Dargestellte zusammenfassen? Zunächst muss festgehalten werden, dass die meisten SchülerInnen mit digitalen Medien aufwachsen und sie als Teil ihres alltäglichen Lebens wahrnehmen und nutzen. Die Schule darf diese Tatsache nicht ignorieren. Die Aufgabe der Schule besteht darin, den analogen Unterricht nach Bedarf mit digitalen zu ergänzen, um Lernprozesse zu optimieren und die Qualität der Inhalte zu steigern. Hierbei können sich unterschiedliche Faktoren hemmend auf die Durchdringung durch neue Medien im Unterricht auswirken. Trotz der Bemühungen seitens der Kultusministerkonferenz gibt es weiterhin einige Problemfelder, die noch nicht ausreichend erforscht wurden.

Die Problematik lässt sich auch außerhalb Deutschlands finden. In der Schweiz beschäftigt sich Dominik Petko mit der Frage, warum Computer und Internet verhältnismäßig selten im Unterricht eingesetzt werden, obwohl diese zunehmend zur Verfügung stehen. Zur Klärung der Frage wurden zwei Modelle herangezogen: das Will/Skill/Tool- sowie das Access/ Competence/Motivation-Modell (Petko 2012: 29). Beide Modelle haben gemeinsam, dass 1. die positive Einstellung der Lehrkraft gegenüber dem Einsatz von Computertechnologie, 2. gute Fähigkeiten im Umgang mit der Technologie und 3. der ausreichende Zugang zu den nötigen Geräten in der Schule, aber auch zu Hause, für einen hohen Einsatz von digitalen Inhalten im Unterricht führt.

Die Faktoren der beiden Modelle sollen anhand der Daten einer repräsentativen Schweizer Bestandsaufnahme von Barras und Petko (2007) untersucht werden. Die Studie wurde durchgeführt, nachdem Schweizer Schulen umfassend modernisiert wurden. Im Rahmen der Modernisierung, die in den Jahren 2002 bis 2007 durchgeführt wurde, erhielt fast jede Schule 
einen kostenlosen Breitband-Internetanschluss und eine Vergünstigung für die Anschaffung von Hard- und Software. Mehr als 1.700 LehrerInnen wurden zu ExpertInnen ausgebildet und etwa 8.000 LehrerInnen besuchten Kurse zur Förderung des Einsatzes der digitalen Medien im Unterricht. Für die Umfrage wurden durch Zufallsstichprobe 1.444 Schulen genommen. Von den 25.214 LehrerInnen, die an diesen Schulen arbeiten, wurden 4.328 Lehrpersonen zufällig ermittelt und gebeten, einen Papierfragebogen auszufüllen. Von 2.044 LehrerInnen haben die Forscher schließlich die beantworteten Bögen zurückerhalten. Erhoben wurden die Daten zu mehreren Aspekten bzgl. des Einsatzes von digitalen Medien: 1. Die Nutzung digitaler Medien im Unterricht: wie und für welche Sozialformen werden digitale Medien eingesetzt? 2. Die technische Infrastruktur: Wie gut ist die Schule mit Computern ausgestattet? 3. Die Kompetenzen der Lehrpersonen: Wie schätzen sich LehrerInnen selbst ein? 4. Die Überzeugungen der Lehrpersonen: Motivation und Einstellungen von Lehrpersonen zu einer intensiven Nutzung digitaler Medien.

Nach der Auswertung der Ergebnisse kommt Petko zum folgenden Schluss: „Gute Infrastruktur, ausreichende Kompetenzen und förderliche Einstellungen sind wesentliche Bedingungen für eine erhöhte Nutzungsfrequenz von ICT [information and communications technology - E.K.] im Unterricht, ohne dass sich diese aus den genannten Faktoren jedoch vollständig erklären ließe“ (2012: 46). Das Resultat der Studie bestätigt die Annahme, dass der Einsatz digitaler Medien mehr benötigt als eine gut ausgestattete Schule und gleichzeitig daran scheitern kann, wenn einer der drei oben herausgearbeiteten Faktoren nicht erfüllt wird. Motivation, Fähigkeiten und Ausstattung stehen stehts in einer Wechselwirkung zu einander.

Im Fokus meiner Forschung befinden sich ebenfalls die Faktoren Zugangsmöglichkeiten, Kompetenzen und Motivation der Lehrkraft in Bezug auf den Einsatz digitaler Medien. Zwar ist mein Forschungsfeld vergleichsweise klein und stark eingeschränkt, doch kann ich durch die Beobachtung 
des Unterrichts und Interviews mit den Lehrkräften, was bei Petko nicht gegeben war, die Wechselwirkung der drei Faktoren deutlicher herausarbeiten.

\section{Empirische Untersuchung an einer Gesamtschule}

Die bis hierhin erarbeiteten Thesen zum Einsatz digitaler Medien im (Russisch-)Unterricht wurden im Rahmen eines Forschungsprojektes während eines halbjährigen Praxissemesters untersucht. Das Ziel der Untersuchung ist der Gewinn neuer Erkenntnisse in Bezug auf die Wechselwirkung zwischen der technologischen Ausstattung der Schule, den Fähigkeiten und der Motivation der Russischlehrkräfte.

\subsection{Rahmenbedingungen der Untersuchung}

Die vorliegende Untersuchung fand im Januar 2018 an einer Gesamtschule in Nordrhein-Westfalen statt. Im Fokus der Studie stand der Russischunterricht von zwei Lehrpersonen. Aufgrund des Datenschutzes werden die Lehrkräfte mit Lehrperson A und Lehrperson B benannt. Beide Lehrkräfte unterrichten seit mehreren Jahren das Fach Russisch von der 8. bis zur 11. Klasse. Im Untersuchungszeitraum unterrichtete die Lehrperson A drei Russischkurse, die Lehrperson B nur einen. Im Unterricht werden die Lehrbücher „Dialog 1“ (2016) und „Dialog 2“ (2017) sowie die dazugehörigen Arbeitshefte eingesetzt. Beiden Lehrwerken ist ein digitaler Unterrichtsmanager auf einer CD-ROM beigelegt. Dieser umfasst eine digitalisierte Version des Lehrwerks sowie digitales Begleitmaterial wie z.B. Hörspiele und Dialogaufnahmen. Der Unterrichtsmanager wurde auf dem 
Schulcomputer im Lehrerzimmer installiert, sodass Lehrkräfte den Unterricht auch in der Schule vorbereiten können. Des Weiteren ist das Programm auch auf den PCs installiert, die mit dem Smartboard verbunden sind. Das bedeutet, dass der Unterrichtsmanager in den Räumen, die über ein Smartboard verfügen, ohne weitere Vorbereitung eingesetzt werden kann. In anderen Räumen muss eine mobile Station mit Laptop samt Videoprojektor erst in die Klasse gebracht werden, falls man dort den Unterrichtsmanager nutzen möchte.

Die Untersuchung lief in zwei Schritten ab. Zuerst wurden die Lehrkräfte zwei Wochen lang im Unterricht beobachtet und der Einsatz der Medien im Stil einer ethnographischen Beobachtung ${ }^{2}$ protokolliert. Auch wussten die Lehrkräfte nicht, welche konkreten Eigenschaften des Unterrichtes ich beobachte und bewerte. Im zweiten Schritt wurden die Lehrkräfte gebeten in einem Leitfadeninterview ${ }^{3}$ ihre Meinung zum Einsatz digitaler Medien im persönlichen und schulischen Umfeld abzugeben. Die Lehrkräfte wurden einen Tag vor dem persönlichen Gespräch über das Thema des Interviews in Kenntnis gesetzt; die Leitfragen haben sie jedoch nicht vorher erhalten. Damit sollte verhindert werden, dass die Lehrkräfte das eigene Verhalten und ihre Einstellung zu Medien bereits vor dem Gespräch reflektieren können.

\footnotetext{
${ }^{2}$ Unter ethnographischer Beobachtung versteht man das Sammeln von Information durch ein temporäres Eintauchen in das Forschungsfeld und das Einnehmen der Perspektive des zu erforschenden Subjekts bzw. Gruppe von Subjekten (vgl. Schulz 2014). Die Protokollierung ähnelt eher einem narrativen Beschreiben der Situation und nicht einer Benennung und Kategorisierung der auftretenden Merkmale.

${ }^{3}$ Ein Leitfadeninterview ist eine qualitative Interviewform mit Fokus auf ein thematisch klares Ziel, jedoch mit Möglichkeit zur Abweichung von der Kernfrage (vgl. Riesmeyer 2011).
} 


\subsection{Ethnographische Beobachtung des Russischunterrichtes}

\section{Lehrkraft A}

In dem Untersuchungszeitraum habe ich die Lehrperson A in den Klassen 8 und 11 begleitet. Während meiner Anwesenheit in der Jahrgangstufe 8 wurde das Themenfeld „Moja sem'ja/Meine Familie“ behandelt. Als zentrales Medium wurde das Werk Dialog 1 (Adler et al., 2016) verwendet, welches für das Thema benötigte Lehr- und Lernmaterial bereitstellt. Um die Reihe erfolgreich abzuschließen, mussten die SchülerInnen den Stammbaum der eigenen Familie auf einem Plakat festhalten und dieses der Klasse präsentieren. Für die beispielhafte Darstellung eines Stammbaumes hat die Lehrkraft A Tafel und Kreide benutzt. Am Beispiel der Familie der Lehrkraft wurde neues Vokabular erarbeitet. Das Erstellen des eigenen Stammbaumes von den SchülerInnen hat mehrere Unterrichtsstunden in Anspruch genommen. Lehrkraft A hat die SchülerInnen gebeten, nach Möglichkeit Fotos von der Familie mitzubringen, um so die Präsentation gehaltvoller gestalten zu können. Leider wurde diese Möglichkeit so gut wie gar nicht genutzt. Im Laufe der Unterrichtsbeobachtung hat die Lehrkraft A zu keinem Zeitpunkt neue Medien eingesetzt.

In der Jahrgangsstufe 11 war das Thema der Unterrichtsreihe „Prazdniki/Feiertage". Hier war das Werk Dialog 2 (Albert et al., 2017) als zentrales Lehr- und Lernmedium im Einsatz. Die SchülerInnen sollten im Verlauf der Reihe unter anderem Einladungen schreiben, Einladungskarten erstellen und auf Einladungen der anderen SchülerInnen antworten. Auch während dieser Unterrichtsreihe hat die Lehrkraft A keine digitalen Medien eingesetzt.

Die Klassenräume der jeweiligen Russischkurse der Lehrperson A verfügten über keine vorinstallierten Geräte, die einen reibungslosen Einsatz neuer Medien hätten ermöglichen können. Das heißt, dass die Lehrperson 
Geräte wie Laptop, Videoprojektor oder CD-Spieler vor dem Unterricht reservieren und zum Unterricht hätte mitbringen müssen. Auch verfügten die Räume über keinen Zugang zum Internet, wodurch nur die Nutzung des mobilen Internets über ein Smartphone o.Ä. möglich wäre. Daher hätte die Lehrkraft alle digitalen Medien vorab für die jeweiligen Geräte bereitstellen müssen.

Für die Russischkurse bestand die Möglichkeit themenspezifische, digitale Inhalte aus dem Unterrichtsmanager des Lehrwerks einzusetzen. Das heißt, dass für den Unterricht geeignete Materialen wie zum Beispiel Hörverstehen-Übungen zur Verfügung standen. Da die Ausstattung der Klassenräume jedoch sehr eingeschränkt war, erforderte der Einsatz neuer Medien zusätzliche Arbeit von der Lehrkraft. Die Faktoren Fähigkeiten und Motivation der Lehrkraft A werden in den nächsten Kapiteln erarbeitet.

\section{Lehrkraft $B$}

Im Kontrast dazu stehen die Ergebnisse der Beobachtung von dem Unterricht, der von der Lehrperson B durchgeführt wurde. Der Klassenraum, in dem diese Lehrkraft den Russischunterricht gehalten hat, verfügte über ein Smartboard und einen Computer mit einem Internetanschluss. Der Unterrichtsmanager des Lehrwerks Dialog 1 war auf dem Computer installiert.

In dem Zeitraum der Beobachtung hat die Lehrkraft B stets mit Hilfe des Smartboards auf digitaler Tafelfläche geschrieben. Wurde die digitale Seite vollgeschrieben, hat die Lehrkraft das Geschriebene nicht gelöscht, sondern an der Seite des Textverarbeitungsprogramms abgelegt bzw. gespeichert, sodass auf diesen Inhalt auch später zurückgegriffen werden konnte. Am Ende der Stunden hat die Lehrkraft die Tafelbilder nicht gespeichert, was aus meiner Sicht sinnvoll gewesen wäre, um beispielsweise in der Klausurvorbereitung bereits besprochene Inhalte erneut anzeigen zu können. 
In den Unterrichtsphasen, in denen mit dem Lehrwerk gearbeitet wurde, hat die Lehrkraft den Unterrichtsmanager bedient, damit alle SchülerInnen sehen konnten, auf welcher Seite bzw. an welcher Aufgabe gearbeitet wird. Dies hatte den Vorteil, dass der Fokus der SchülerInnen aufrecht erhalten blieb und niemand erst die richtigen Stellen suchen musste. Wenn Texte aus dem Buch vorgelesen wurden, konnten die SchülerInnen zum Smartboard gehen und unbekannte Lexik farblich markieren. Übersetzungen wurden direkt an die unbekannten Wörter geschrieben. Auch hat die Lehrkraft B die im Lehrwerk abgebildeten Tabellen oder Mindmaps ohne Umwege auf den digitalen Seiten des Lehrwerks vervollständigen lassen. Dadurch konnte nicht nur Zeit gespart werden, indem größere Übergänge zwischen verschiedenen Medien und Werkzeugen verhindert wurden, sondern es wurde auch ein unmittelbarer Einstieg in die Aufgabenbearbeitung ermöglicht.

Des Weiteren hat die Lehrkraft B ihren Kurs mit Audio- und Video-Dateien, die im Unterrichtsmanager zur Verfügung stehen, bereichert. Natürlich hätte die Lehrkraft B die meisten dieser Aufgaben auch selbstständig vortragen können. Während meiner Unterrichtsbeobachtung wurden nur wenige externe Medien eingesetzt. Wenn sie eingesetzt wurden, dann oft von den SchülerInnen an ihren Smartphones. So konnte ich beobachten, wie die SchülerInnen unbekanntes Vokabular recherchierten.

Problematisch dagegen ist mir der Übergang von einzelnen Arbeitsphasen aufgefallen, wenn beispielsweise neue Dateien, Ansichten oder Werkzeuge am Smartboard geöffnet bzw. geladen werden mussten. Ich stellte fest, dass die SchülerInnen immer dann dem Unterrichtsgeschehen nicht mehr konzentriert folgten, wenn die Technik streikte oder die Lehrkraft nicht die gewünschten Funktionen fand. Störungen, die daraus resultierten, sind darauf zurückzuführen, dass der Einsatz digitaler Werkzeuge nicht reibungslos mit dem analogen Unterricht verzahnt ist und daher eine Steigerung der potentiellen Unruhen stattfindet. 


\subsection{Das Leitfadeninterview mit Russischlehrkräften}

Im zweiten Schritt der Untersuchung wurde ein Interview mit den Lehrpersonen A und B durchgeführt. Beide Interviews hatten eine durchschnittliche Länge von etwa 20 Minuten. Im Folgenden möchte ich die wichtigsten Ergebnisse präsentieren; die Interpretation der Ergebnisse findet im nächsten Kapitel statt.

Zuerst wurde die Frage nach der privaten Nutzung von Geräten wie Computer, Tablet und Smartphone gestellt. Beide Lehrpersonen gaben an, sie würden alle drei Gerätekategorien regelmäßig nutzen. Auf die Frage, welche davon sie im Russischunterricht nutzen bzw. von den SchülerInnen nutzen lassen, gab die Lehrperson A an, sie benutzt selten einen Computer und öfter das Smartphone, da ihre Klassenräume über keinen Computer verfügen. Zudem kritisierte sie die Abhängigkeit des Unterrichtsmanagers vom Betriebssystem Microsoft Windows, da sie privat Geräte der Firma Apple nutzt. Das führt dazu, dass die Vorbereitung von digitalen Inhalten hauptsächlich am Schulcomputer stattfindet. Lehrperson B nutzt dagegen so gut wie in jeder Stunde einen Computer, ein Smartboard sowie Smartphones. Letztere werden von der Lehrperson B und von den SchülerInnen oft als digitale Lexika eingesetzt. Sie würde Smartphones noch vielfältiger einsetzen, wenn die Schule über einen WLAN-Zugang verfügen würde. Die Lehrperson B erzählte, dass sie seit etwa zwei Jahren das Smartboard benutzt. Früher ist sie oft mit den SchülerInnen in den Computerraum der Schule gegangen, wo sie die kyrillische Schrift installiert hat, damit die SchülerInnen recherchieren und Plakate erstellen konnten.

Im Gegensatz zum privaten Umfeld sehen beide Lehrpersonen die Schule als schlecht ausgestattet an. Sie gaben an, dass die Computer veraltet seien und es davon zu wenige gäbe. Da die Schule über keinen WLAN-Zugang verfügt, haben Lehrkräfte also nur in wenigen Räumen, in denen ein Computer installiert ist, Zugang zum Internet. Auf die Frage, ob die Lehrkräfte 
ihre eigenen Notebooks zur Schule mitbringen würden, antwortete die Lehrperson A mit einem Nein, weil es in der Schule kein WLAN gibt. Die Lehrperson B bringt trotz dieser Umstände ihr Notebook mit, um z.B. Lerninhalte der Stunde vorzubereiten.

Beide Lehrkräfte gaben an, sie würden viel Unterrichtsmaterial im Lehrerzimmer am Computer vorbereiten, diese seien jedoch langsam. „Sogar in Russland ist die Ausstattung besser!", antwortet die Lehrperson B. Auch gäbe es nicht genügend PCs im Lehrerzimmer, um über einen längeren Zeitraum an diesen zu arbeiten, meinten beide Lehrkräfte.

Auf die Frage, ob digitale Medien im Russischunterricht oft eingesetzt werden, antwortete Lehrperson A mit einem ,wenig“ und begründete es mit dem Fehlen der Ausstattung. In einem analogen Klassenraum würde der Einsatz digitaler Medien einen größeren Aufwand in der Vorbereitung bedeuten. Die Lehrperson B antwortet mit ,Ja, zum Beispiel zeige ich auch oft Videos". Sie betonte, wie wichtig es sei, die Sprache auch visuell wahrzunehmen, weil dies das Lernen erleichtere. Vor allem russische Zeichentrickfilme und Musikvideos können bei den SchülerInnen Spaß am bzw. Motivation für das Lernen hervorrufen.

Offiziell herrscht an der Schule ein Smartphone-Verbot im Unterricht und in den Pausen. Die SchülerInnen missachten dieses jedoch. Konsequenzen für Verstöße gab es nur in seltenen Fällen. Auf die Frage, ob solch ein Verbot sinnvoll ist, antwortete Lehrperson A mit „Ja, und ich habe versucht es durchzusetzen, aber du machst dich eher lächerlich, weil du ständig am Ermahnen und Hinterherlaufen bist". Sie würde es begrüßen, wenn alle SchülerInnen ihre Geräte am Schuleingang abgeben müssten. Lehrperson B, die sich als technikfreundlicher gezeigt hat, hält nichts von einem Smartphone-Verbot. Sie geht davon aus, dass die SchülerInnen sich nicht daran halten würden. Und außerdem sei das Gerät vielseitig einsetzbar, auch im Unterricht. Die Frage zum konkreten Einsatz von Smartphones im Unterricht beantworteten die Lehrkräfte ähnlich: Beide haben

Anastasia Drackert und Katrin Bente Karl (Hg.), Didaktik der slawischen Sprachen

Beiträge zum 2. Arbeitskreis in Innsbruck (19.02.-20.02.2018)

(C) 2019 innsbruck university press, ISBN 978-3-903187-80-1, DOI 10.15203/3187-80-1 
bereits Smartphones benutzt, um Kurzfilme im Russischunterricht zu erstellen. Lehrperson A erwähnte, dass durch das selbstständige und kreative Arbeiten mit Technik vor allem die Motivation der SchülerInnen gesteigert werden konnte.

Beide Lehrpersonen waren sich darüber einig, dass für den Unterricht die Schule lieber mit Tablets als mit stationären PCs oder Notebooks ausgestattet werden sollte, da diese flexibler einsetzbar sind. Damit würden die SchülerInnen einen unkomplizierten und ihnen bekannten Zugang zu digitalen Inhalten erhalten. Die Idee im Unterricht komplett auf Papier zu verzichten, was bereits an manchen Schulen praktiziert wird, halten beide der befragten Lehrkräfte für wenig sinnvoll. „Nein, das wäre grauenvoll! Aber beides zu haben, das wäre am besten" äußerte sich Lehrperson B.

Ein weiteres Themenfeld war die Einschätzung der Medienkompetenz der KollegInnen und der SchülerInnen. Beide gaben an, dass viele Lehrkräfte nicht auf dem aktuellen Stand seien und junge ReferendarInnen die Technik besser beherrschen würden. Jedoch würden auch sie während ihrer Lehramtsausbildung nicht alle nötigen Kenntnisse beigebracht bekommen.

Aus der Sicht der Lehrpersonen sind die meisten SchülerInnen gut geschult im Umgang mit technischen Geräten. Ein Informatikkurs der Schule bereitet die SchülerInnen bereits früh auf die Bedienung eines Computers vor. Jedoch fehlt den SchülerInnen ein verantwortungsvoller und reflektierter Umgang mit digitalen Medien. „Sie glauben alles und das ist das Problem!“" äußerte sich Lehrperson B. Beide Lehrkräfte sehen noch Defizite in der Sensibilisierung und wünschen sich mehr Unterrichtszeit, die diese Lücke schließen soll. Doch während Lehrperson A glaubt, man könnte das Problem eher im Informatikunterricht angehen, fordert Lehrperson B, dass die Schulung fächerübergreifend stattfinden soll: „,Wenn es nicht mit dem Fach zu tun hat, ist es für SchülerInnen nicht interessant." 
Ihrer Meinung nach muss die Förderung der Medienkompetenz realitätsnah und unterrichtsbezogen erfolgen.

Auf die Frage, ob die anstehende Digitalisierung an deutschen Schulen sinnvoll und notwendig ist, antworteten beide Lehrkräfte mit einem eindeutigen , $J a^{\prime}$ ", wobei Lehrperson B hinzufügte, dass sie nicht an den Erfolg des Förderungsprogramms glaubt. Zwar würden sich die Lehrkräfte den Ausbau der digitalen Infrastruktur wünschen und diesen Schritt unterstützen, doch scheuen sich die zuständigen Instanzen auf der Schulebene vor dem Arbeitsaufwand, den dieser Prozess mit sich bringen würde.

Anschließend äußerten die Befragten ihre Wünsche und Sorgen: Lehrperson A wünscht sich, dass die Lehramtsausbildung an das digitale Zeitalter angepasst wird und die Lehrpläne diesbezüglich überarbeitet werden. Darüber hinaus sollte jeder Raum über eine gute Ausstattung verfügen, für die jeweils eine Lehrperson zuständig ist, damit alles an diese angepasst wird. Somit wäre jede Lehrkraft für die Funktion der eigenen Geräte verantwortlich und könnte alle Lerninhalte auf einem Gerät speichern.

Lehrperson B erklärte, dass der Medieneinsatz viel zusätzliche Zeit in der Vorbereitung kostet, die LehrerInnen aber über keine Zeitreserven verfügen. Die Lehrkraft wünscht sich, dass dies bei der Erstellung von Kernlehrund Stundenplänen stärker berücksichtigt wird.

\subsection{Interpretation der Forschungsergebnisse}

Nun möchte ich die Ergebnisse aus den Beobachtungen während des Russischunterrichts den Aussagen der Lehrkräfte aus den Interviews gegenüberstellen. 
Nach zwei Wochen Beobachtung des Unterrichts der Lehrperson A musste ich feststellen, dass diese keine digitalen Medien in ihren beiden Russischkursen eingesetzt hat, obwohl digitalisiertes Unterrichtsmaterial zur Verfügung stand. Es wurde festgestellt, dass die Ausstattung der Räume nicht für digitales Lehren vorgesehen war und die Lehrperson somit für jede Stunde erst Geräte hätte herbeischaffen müssen. Es wäre daher falsch anzunehmen, dass die Lehrperson entweder nicht über die nötigen medialen Kenntnisse oder nicht ausreichende Motivation verfügte, denn mehrere Argumente sprechen dagegen. Zunächst zeigte sich die Lehrperson A im persönlichen Gespräch als durchaus technik- und medieninteressiert. So gab sie an, die Stundenplanung und Erstellung des Lehrmaterials am PC bzw. Mac durchzuführen. Sie betonte, dass gute technische Ausstattung in jedem Klassenraum wünschenswert sei. Auch begrüßte sie die Pläne des Schulministeriums, umfassende Digitalisierungsmaßnahmen an deutschen Schulen vorzunehmen. Außerdem konnte ich meine Ergebnisse aus dem Russischunterricht mit anderen Fächern, die die Lehrkraft A unterrichtete, vergleichen. So hat sie in dem Fach Gesellschaftslehre im selben Untersuchungszeitraum mehrmals neue Medien eingesetzt: Es wurden historische Filme gezeigt und analysiert, digitales Kartenmaterial wurde zum besseren Verständnis hinzugezogen und anschließend (analog) bearbeitet und digitalisierte, historische Quellen wurden interpretiert. Nun muss man aber auch erwähnen, dass die Lehrräume dieser Fächer zum Teil besser ausgestattet waren. So befand sich im Klassenraum, in dem Geschichte für die Oberstufe unterrichtet wurde, ein PC sowie ein Beamer. Es konnten keine Schwierigkeiten bei der Bedienung der Geräte beobachtet werden. Daher kann in diesem Fall die Behauptung aufgestellt werden, dass der Faktor Ausstattung sich hemmend auf die Faktoren Motivation und Fähigkeiten der Lehrkraft auf den Einsatz von Medien auswirkt.

Anders ist die Situation bei der Lehrperson B, deren Russischunterricht stets in einem medial gut ausgestatten Klassenraum stattgefunden hat. Diese Lehrkraft zeigte ihre Technikaffinität nicht nur im persönlichen Gespräch abseits des Klassenraumes, sondern nutzte tatsächlich durchgehend 
digitalisierte Inhalte und elektronische Hilfsmittel im Unterricht. In der Beobachtungsphase wurde festgestellt, dass die Lehrkraft das vorhandene Smartboard in jeder Unterrichtsphase einsetzte. Das Smartboard war daher nicht nur ein moderner Tafelersatz, sondern eine Bereicherung möglicher Unterrichtsmethoden und -inhalte. Im Interview konnte dieser Eindruck bestätigt werden. Lehrperson B zeigte sich stets interessiert an neuen Medien und ihren Funktionen im Unterricht. Im Unterschied zu vielen KollegInnen sprach sich die Lehrkraft B gegen ein Smartphone-Verbot an ihrer Schule aus. Sie sieht in erster Linie eine mögliche Bereicherung des Unterrichts durch die sinnvolle Nutzung mobiler Geräte. Betrachtet man nun die Ergebnisse vor dem Hintergrund der Forschung von Petko (2012), so kann behauptet werden, dass sich die Faktoren Motivation, Fähigkeiten und Ausstattung tatsächlich gegenseitig positiv verstärken. Ich möchte jedoch darauf hinweisen, dass sich die vorliegenden Forschungsergebnisse nur auf eine einzige Schule beziehen und dementsprechend keine Schlussfolgerung auf die Situation in NRW oder gar Deutschland erlauben.

\section{Resümee: Digitale Medien im Schulalltag}

Ziel dieser Arbeit war die Erforschung des Einsatzes digitaler Medien in der Schulpraxis und die Herausarbeitung hemmender und förderlicher Faktoren. Die von Petko (2012) postulierte Wechselwirkung von Motivation und Fähigkeiten der Lehrkräfte sowie Ausstattung der Schulen in Bezug auf den Grad des Medieneinsatzes konnte auch in dem untersuchten Russischunterricht festgestellt werden. Petkos Annahmen, die er nach der Auswertung der Schweizer Studie aufgestellt hat, können hier bestätigt werden. Zwar ist es auch mir nicht gelungen, eine genaue Gewichtung und Einflussnahme der einzelnen Faktoren auszuarbeiten, doch liegt es an dem begrenzten Forschungsraum, den ich in der Praxis vorgefunden habe. 
Durch die Zweiteilung des Projektes in ethnographische Beobachtung auf der einen und dem Leitfadeninterview auf der anderen Seite konnte ich die Selbsteinschätzung der Lehrkräfte ihrer Unterrichtsgestaltung gegenüberstellen. Dabei komme ich zum folgenden Schluss: Lehrperson A sieht in digitalen Medien einerseits eine Bereicherung für den Unterricht, andererseits eine große Hürde in der Vorbereitung. Dabei könnte sie von den mobilen Videoprojektoren Gebrauch machen oder die Audio-Aufgaben des Unterrichtsmanagers auch mit Hilfe des Smartphones und eines mobilen Lautsprechers im Unterricht einsetzen. Bei dieser Lehrkraft wirkt sich eine mangelnde Ausstattung negativ auf die Frequenz des Einsatzes von neuen Medien aus. Ihr persönliches Engagement genügt nicht, um diesem Faktor entgegenzuwirken.

Lehrperson B kann als engagiert und technikaffin charakterisiert werden. Sie nutzt die vorhandene Ausstattung vielseitig und bereichert damit ihren Unterricht. In diesem Kurs kommen die drei Kernfaktoren - Motivation, Fähigkeiten und Ausstattung - zusammen und ermöglichen einen authentischen und abwechslungsreichen Input, vom dem die SchülerInnen profitieren können.

Zusammenfassend kann ich folgendes Fazit aus der Forschungsarbeit ziehen: Der Einsatz digitaler Medien im Schulunterricht ist kein sich selbstantreibender Prozess. Es ist ein Vorgang, der von Lehrkräften nicht nur Kompetenzen, sondern auch persönliches Interesse, Engagement und Motivation erfordert. Dennoch bleibt die Ausstattung der Schule ein enorm wichtiger Faktor. Erst wenn die technologischen Möglichkeiten gegeben sind, können Lehrkräfte sich mit diesen im beruflichen Umfeld vertraut machen und diese im Unterricht einsetzen.

Abschließend bleibt zu sagen, dass digitale Medien einen deutlichen Mehrwert für den Russischunterricht bringen können. Ihre Auswahl und Einsatz müssen jedoch an mehrere Faktoren geknüpft werden. So soll stets die Notwendigkeit hinterfragt werden, denn Medien sind nur ein weiteres 
Werkzeug in der Methodenkiste der Lehrkräfte. Auch digitale Inhalte müssen im Sinne der Merkmale des guten Unterrichts vorbereitet werden. Erst wenn digitale Medien sich mit dem analogen Unterricht optimal verzahnen, kann ein guter Unterricht gelingen.

\section{Vorschläge zum sinnvollen Medieneinsatz im Russisch- unterricht bei der Berücksichtigung nicht ausreichender schulischer Ausstattung}

Zuletzt möchte ich einige eigene Ideen zur Verbesserung des Medieneinsatzes bei unzureichender Ausstattung der Schulräume präsentieren. Die Studie hat ergeben, dass eine unzureichende Ausstattung der Schulräume - verständlicherweise - für den Einsatz von digitalen Medien im Unterricht hemmend sein kann. Daher wurden praxisnah Überlegungen angestellt, wie bereits vorhandene Ressourcen genutzt werden können, um den Unterricht sinnvoll zu bereichern.

Die mehrmonatige Praxisphase hat gezeigt, dass die meisten SchülerInnen ein Smartphone und manchmal sogar ein Tablet in der Schule mitführen. In vielen Schulen sind Smartphones nach wie vor auf dem Schulgelände nicht erlaubt - so auch in der hier untersuchten Gesamtschule. Somit verbleiben die Möglichkeiten des World-Wide-Webs in Bezug auf das Lernen im schulischen Kontext ungenutzt in der Hosentasche, denn trotz des Verbots nutzen viele SchülerInnen ihre Geräte, nur eben heimlich. Meiner Meinung nach stellt diese Tatsache eine falsche Entwicklung dar, denn Medienkompetenz bedeutet nicht nur, dass die SchülerInnen den kritischen Umgang mit digitalen Medien erlernen sollen; auch der verantwortungsvolle Umgang mit Geräten, die den Zugang ermöglichen, muss den SchülerInnen vermittelt werden. SchülerInnen könnten ihre Smartphones

Anastasia Drackert und Katrin Bente Karl (Hg.), Didaktik der slawischen Sprachen

Beiträge zum 2. Arbeitskreis in Innsbruck (19.02.-20.02.2018)

(C) 2019 innsbruck university press, ISBN 978-3-903187-80-1, DOI 10.15203/3187-80-1 
und Tablets für diverse unterrichtliche Zwecke einsetzen, wenn die Schulausstattung den Bedarf nicht decken kann. So ergeben sich viele Aufgabenbereiche, die mit den mobilen Endgeräten bearbeitet werden können:

Lexikon und Wortschatzarbeit: Auch ohne einen mobilen Internetzugang können Smartphones als mobile Wörterbücher dienen. SchülerInnen, die stets nach neuem Vokabular fragen, können in diversen Situationen selbstständig unbekannte Lexik in vorinstallierten Applikationen nachschlagen. Die Verantwortung für das Erlenen neuen Vokabulars wird ein Stück weit den SchülerInnen überlassen. Zudem kann ein Arrangement der Lehrkraft mit den SchülerInnen dafür Sorge tragen, dass nachgeschlagene Lexik markiert und zum Ende der Stunde der Lerngruppe präsentiert wird.

Recherche/Umgang mit Heterogenität: Mobile Endgeräte lassen sich in diversen Situationen zu Recherchezwecken einsetzen. Bei aufkommenden Fragen zur russischen Kultur, Geografie, Gesellschaft u. ̈̈. kann die Lehrkraft gezielt an die SchülerInnen binnendifferenzierte Arbeitsaufträge stellen, die am besten mit der Hilfe von russischen Suchmaschinen bearbeitet werden können. Einerseits kann dieses Vorgehen den Redeanteil der Lehrkraft senken und diesen an die SchülerInnen umleiten, andererseits erfordern derart spontane Erarbeitungs- und anschließende Präsentationsphasen ein hohes Maß an Flexibilität seitens der Lehrkraft. Dennoch können so die SchülerInnen die bereits vorhandenen Ressourcen nutzen, um die Antworten auf ihre Fragen selbstständig zu erarbeiten.

Konsummedien: Unter diesem Punkt werden digitale Medien zusammengefasst, die primär dem Konsum dienen, sich aber sinnvoll im Fremdsprachenunterricht als authentisches Material einsetzen lassen. Konkret würde das heißen, dass man Musik(-videos), Blog- und Foreneinträge, Beiträge aus sozialen Medien u. ̈. im Klassenraum als ein weiteres Mittel zur Vermittlung der Sprache und Kultur nutzt. Das digitale Arbeitsmaterial lässt sich vielfältig einsetzen: Moderne Lieder können übersetzt, nachgesungen und analysiert werden; Blogs können nach Interessen verfolgt und im Kurs 
präsentiert werden. Die oft durchgeführte Unterrichtsreihe zum Thema „Meine Vorbilder“" kann mit Hilfe von sozialen Medien unter neuem Blickwinkel durchgeführt werden, z.B. Erfüllung der Vorbildfunktion von Sportlern, Künstlern. Gleichzeitig würden die SchülerInnen den kritischen Umgang mit unterhaltenden und meinungsteilenden Medien lernen. Vor allem scheinen digitale Blogeinträge viele Vorteile für die interkulturelle Kompetenz zu bieten (Plikat 2010: $53 \mathrm{ff}$ ). Neben den bereits angesprochenen Aspekten Authentizität und Aktualität ermöglichen Blogs auch interaktives Handeln, wenn LeserInnen mit AutorInnen über E-Mail oder Kommentarfunktion in Kontakt treten können.

Unterrichtsmanager des Lehrwerks: Modernen Schulbüchern wird oft eine digitalisierte Version des Lehrwerks auf einem Datenträger beigelegt. So auch bei den Werken Dialog 1 (2016) und Dialog 2 (2017). Dabei handelt es sich nicht nur um eine reine Kopie des Buches in einem digitalen Format, sondern auch um Aufgaben und Medien, die an einem PC bearbeitet werden können. In der oben dargelegten Untersuchung wurde gezeigt, wie Lehrkräfte mit dem Unterrichtsmanager an einem Smartboard arbeiten können. Doch nicht für alle Inhalte wird diese Ausstattung verlangt. So lassen sich beispielsweise Aufnahmen wie Lieder, Dialoge und Erzählungen vom Datenträger bzw. PC auch auf ein mobiles Endgerät übertragen. In Kombination mit einem kleinen, mobilen Lautsprecher können Audiodateien laut genug abgespielt werden. Natürlich könnte auch die Lehrkraft einen großen Teil von Liedern und Texten selbst vortragen, doch gewinnt der Unterricht an Abwechslung und Qualität, wenn die SchülerInnen unterschiedliche HerkunftssprecherInnen und authentische - wenn auch didaktisierte - Materialien zu hören bekommen.

Doch nicht immer sind elektronische Geräte im Unterricht nötig, um mit digitalen Medien sinnvoll arbeiten zu können. Viele Aufgaben können aus dem schulischen Lernraum ausgelagert werden und lassen sich beispielsweise als Hausaufgabe erledigen und können anschließend im Unterricht besprochen werden. SchülerInnen können am heimischen PC Blogeinträge

Anastasia Drackert und Katrin Bente Karl (Hg.), Didaktik der slawischen Sprachen

Beiträge zum 2. Arbeitskreis in Innsbruck (19.02.-20.02.2018)

(C) 2019 innsbruck university press, ISBN 978-3-903187-80-1, DOI 10.15203/3187-80-1 
lesen, übersetzen oder selbstständig eigene Einträge erstellen. Auch könnten zu Hause russische Unterhaltungsmedien konsumiert werden und Lerninhalte recherchiert werden. Fortgeschrittene Gruppen können sogar mit russischen Lernvideos arbeiten oder ihre eigenen erstellen, die von der Lehrkraft im nächsten Lernjahr genutzt werden können. Vorstellbar wäre das Erstellen eigener Mini-Serien, wie z.B. Nachrichten- und Kochsendungen mit Russlandbezug. Der Schaffensprozess ermöglicht den SchülerInnen eine komplexere Auseinandersetzung mit digitalen Medien, die beim reinen Konsumieren bzw. Bearbeiten sicherlich so nicht gegeben ist.

Die ausgewählten Vorschläge sollen als Anregung und Motivation dienen, digitale Medien im Unterricht einzusetzen. Sie sind nur ein kleiner Teil der umfangreichen Medienwelt, die sich stets weiterentwickelt und daher neue Herangehensweisen und Unterrichtsmethoden ermöglicht. Natürlich setzt der Gebrauch digitaler Medien im Unterricht voraus, dass diese gründlich aufgearbeitet und sinnvoll in den Unterrichtskontext integriert werden. Doch wenn Lehrkräfte sich diese Kompetenzen angeeignet haben, steht ihnen ein vielseitiges Werkzeug und eine unerschöpfliche Quelle an Möglichkeiten der Unterrichtsgestaltung zur Verfügung.

\section{Literatur}

Adler, I., Boiselle, T., Breitsprecher, R., Chwoika, A., Heller, M., Müller, J., Nadchuk, E., Seidel, A., Semashkina, L. \& Steinbach, A. (2016). Dialog 1. Schülerbuch für den Russischunterricht. Berlin: Cornelsen.

Albert, A., Adler, I., Boiselle, T., Breitsprecher, R., Chwoika, A., Müller, J., Nadchuk, E., Seidel, A. \& Steinbach, A. (2017). Dialog 2. Schülerbuch für den Russischunterricht. Berlin: Cornelsen. 
Biermann, R. (2009). Der mediale Habitus von Lehramtsstudierenden. Eine quantitative Studie zum Medienverhalten angehender Lehrpersonen. Wiesbaden: VS Verlag für Sozialwissenschaften.

Bleckmann, P. (2016). Statement $» M e d i e n m u ̈ n d i g k e i t-w e l c h e r$ Weg führt zum Ziel? «, öffentliche Diskussionsveranstaltung im Bundestag zur Vorstellung des TAB-Gutachtens »Elektronische Medien und Suchtverhalten " Verfügbar unter: www.alanus.edu/fileadmin/downloads/fachbereiche_und_studienanbegote/fb_bildungswissenschaft/fachbereich/MeMue_Beitrag_Bleckmann.pdf [28.08.2018].

Grafe, S., Herzig, B. \& Tulodziecki, G. (2010). Medienbildung in Schule und Unterricht. Grundlagen und Beispiele. Bad Heilbrunn: Julius Klinkhardt Verlag.

Grünewald, A. (2010). Medien. In C. Surkamp (Hrsg.), Metzler Lexikon (207-210). Fremdsprachendidaktik, Stuttgart: Springer.

Hartmann, S. \& Purz D. (2018). Unterrichten in der digitalen Welt. Göttingen: Vandenhoeck \& Ruprecht.

Kommer, S. (2010). Kompetenz Medienumgang? Eine Qualitative Untersuchung zum medialen Habitus und zur Medienkompetenz von SchülerInnen und Lehramtsstudierenden. Opladen/Farmington Hills: Budrich UniPress.

Kultusministerkonferenz (2012). Medienbildung in der Schule. Verfügbar unter: https://www.kmk.org/fileadmin/Dateien/veroeffentlichungen_beschluesse/2012/2012_03_08_Medienbildung.pdf [27.08.2018].

Lankau, R. (2017). Kein Mensch lernt digital. Über den sinnvollen Einsatz neuer Medien im Unterricht. Weinheim: Beltz.

Ministerium für Schule und Weiterbildung des Landes Nordrhein-Westfalen (Hrsg.). Kernlehrplan für die Sekundarstufe II. Gymnasium/Gesamtschule in Nordrhein-Westfalen. Russisch, 1. Auflage 2014.

Moser, H. (2014). Grundkurs Schulmanagement VIII. Digitale Medien in der Schule. Medienkompetenz für den Unterricht. Kronach: Carl Link.

Anastasia Drackert und Katrin Bente Karl (Hg.), Didaktik der slawischen Sprachen

Beiträge zum 2. Arbeitskreis in Innsbruck (19.02.-20.02.2018)

(C) 2019 innsbruck university press, ISBN 978-3-903187-80-1, DOI 10.15203/3187-80-1 
Petko, D. (2012). Hemmende und förderliche Faktoren des Einsatzes digitaler Medien im Unterricht: Empirische Befunde und forschungsmethodische Probleme. In R. Zander-Schulz, B. Eickelmann, H. Moser, H. Niesyto \& P. Grell (Hrsg.), Jahrbuch Medienpädagogik 9 (2950). Heidelberg: Springer.

Plikat, J. (2010). Desde Cuba con amor: Überlegungen zur Arbeit mit Blogs als Grundlage interkultureller Lernprozesse im fortgeschrittenen Fremdsprachenunterricht. In D. Caspari \& Küster, L. (Hrsg.), Wege zu interkulturellen Kompetenz. Fremdsprachendidaktische Aspekte der Text- und Medienarbeit (51-59). Frankfurt am Main: Peter Lang.

Riesmeyer, C. (2011). Das Leitfadeninterview. Königsweg der qualitativen Journalismusforschung? In O. Jandura, T. Quandt \& J. Vogelgesang (Hrsg.), Methoden der Jouranlismusforschung (223-236). Wiesbaden: Springer.

Schulz, M. (2014). Ethnografische Beobachtung. In: A. Tilmann, A., S. Fleischer \& K.-U. Hugger (Hrsg.), Handbuch Kinder und Medien (225-235). Wiesbaden: Springer.

Spanhel, D. (2001). Medienpädagogische Kompetenz als integraler Bestandteil der Lehrerprofessionalität. In B. Herzig (Hrsg.), Medien machen Schule. Grundlagen, Konzepte und Erfahrungen zur Medienbildung (267-294). Bad Heilbrunn: Julius Klinkhardt Verlag.

Spitzer, M. (2012). Digitale Demenz. München: Droemer Knaur.

Wapenhans, H. (2014). Medieneinsatz im Russischunterricht. In A. Bergmann (Hrsg.), Fachdidaktik Russisch. Eine Einführung (253-265). Tübingen: Narr. 